\title{
25-gauge vitrectomy with gas tamponade for rhegmatogenous retinal detachment: experienced vs. inexperienced surgeons
}

Martin Pencak ( $\square$ pencak@volny.cz)

Department of Ophthalmology, Third Faculty of Medicine, Charles University in Prague and University Hospital Kralovske Vinohrady, Prague https://orcid.org/0000-0002-9090-0998

\section{Miroslav Veith}

Department of Ophthalmology, Third Faculty of Medicine, Charles University in Prague and University Hospital Kralovske Vinohrady, Prague

\section{Zbynek Stranak}

Department of Ophthalmology, Third Faculty of Medicine, Charles University in Prague and University Hospital Kralovske Vinohrady, Prague

\section{Jakub Dite}

Department of Ophthalmology, Third Faculty of Medicine, Charles University in Prague and Universit Hospital Kralovske Vinohrady, Prague

\section{Jana Vranova}

Department of Medical Biophysics and Medical Informatics, Third Faculty of Medicine, Charles University, Prague

\section{Pavel Studeny}

Department of Ophthalmology, Third Faculty of Medicine, Charles University in Prague and University Hospital Kralovske Vinohrady, Prague

\section{Research article}

Keywords: retinal detachment, $25 \mathrm{~g}$ vitrectomy, gas tamponade, inexperienced surgeon

Posted Date: August 26th, 2020

DOl: https://doi.org/10.21203/rs.3.rs-53301/v2

License: (c) (1) This work is licensed under a Creative Commons Attribution 4.0 International License. Read Full License 


\section{Abstract}

Introduction: To compare the results and complication rates of a 25-gauge pars plana vitrectomy (25g PPV) with gas tamponade for rhegmatogenous retinal detachment (RRD) between experienced and inexperienced surgeons.

Materials and Methods: This is a retrospective comparative consecutive case series study of patients with uncomplicated RRD treated with 25g PPV with gas tamponade. Patients were divided into 2 groups: In Group 1 (ESG) the procedure was performed by an experienced vitreoretinal surgeon and in Group 2 (ISG) the procedure was performed by 2 inexperienced surgeons. Anatomical and functional results and complication rates were compared between the two groups.

Results: 216 eyes were included in the study. In the ESG (106 eyes), the single operation success rate was $94.3 \%$, and the final success rate was $100 \%$. The mean best-corrected visual acuity (BCVA) improved from 0.38 decimal to 0.73 decimal. In the ISG (110 eyes), the single operation success rate was $93.6 \%$, and the final success rate was $100.0 \%$. The mean BCVA improved from 0.33 decimal to 0.74 decimal. The differences between groups were not statistically significant. There was no difference in complication rates between groups.

Conclusions: A 25g PPV with gas tamponade for treatment of RRD yields excellent anatomical results and improvement in BCVA. With good technique and use of modern vitrectomy machines and instruments, even inexperienced surgeons can achieve high single operation success rate, suggesting a short learning curve. The complication rate is comparable between experienced and inexperienced surgeons.

\section{Introduction}

Rhegmatogenous retinal detachment (RRD) is a vision-threatening condition that requires prompt surgical intervention. Several surgical techniques for the treatment of RRD have been developed with scleral buckling, pneumatic retinopexy, and pars plana vitrectomy (PPV) being currently used[1-6]. Development of sutureless, small gauge vitrectomy as well as advancements in surgical techniques and equipment have made PPV increasingly popular, among surgeons, for the management of RRD $[7,8]$. In our study, we compared the anatomical success rate, change in visual acuity, and complication rates in patients with RRD, performed using a 25-gauge (25g) PPV with gas tamponade without scleral buckling, between an experienced surgeon and two inexperienced surgeons.

\section{Materials And Methods}

\section{Patient selection}

Ours was a single-center retrospective comparative consecutive case series study. Patients with RRD treated with 25g PPV with gas tamponade without scleral buckling between October 2015 and June 2018 
in the Department of Ophthalmology of the University Hospital Kralovske Vinohrady, Prague, were included in the study. Both pseudophakic and phakic patients were included. We excluded patients with proliferative vitreoretinopathy (PVR) grade $\mathrm{C}$ and higher, patients with a previous perforating eye injury and patients with follow-up periods shorter than 3 months. Patients were then divided into two groups. In the first group (the experienced surgeon group [ESG]) include patients treated by an experienced surgeon (MV, 1909 PPV at the beginning and 2438 PPV at the end of the inclusion period). In the second group (the inexperienced surgeon group [ISG]) included patients treated by two inexperienced surgeons (MP and ZS, 28 and 22 PPV, respectively, at the beginning and 172 and 161 PPV, respectively, at the end of the inclusion period). Both inexperienced surgeons were trained by the same surgeon (MV) and assisted in PPV operations for 2 years prior to their first solo vitrectomy. Both had no previous experience with intraocular surgery. We compared the anatomical success rate, change in visual acuity, and complication rate between groups. All patients signed an informed consent before the surgery. The study protocol adhered to the tenets of the Declaration of Helsinki Principles. Approval was granted by the Ethics Committee of Third Faculty of Medicine, Charles University, Prague and the Kralovske Vinohrady University Hospital, Prague under the number EK-R/01/0/2020.

\section{Pre- and postoperative assessment}

In all patients, the pre- and postoperative best-corrected visual acuity (BCVA) was assessed using ETDRS charts, results of which were then converted to decimal values for statistical analysis. BCVA of counting fingers, hand motion, or light perception were converted do decimal values using the chart published by Holladay[9]. Pre- and postoperative slit-lamp examination and fundus biomicroscopy were performed to determine the extent of RRD, the location and number of retinal brakes (RB), grading of the preoperative PVR, as well as to assess the postoperative state of the retina. Intraocular pressure (IOP) was measured using non-contact tonometry using a NT-530 (Nidek, Aichi, Japan) preoperatively, on the first postoperative day, and on all visits during follow-up. Hypotony was defined as an IOP $<10 \mathrm{mmHg}$ and hypertension as an IOP $>25 \mathrm{mmHg}$. The primary endpoint was the single surgery retinal reattachment rate. The secondary endpoints were the postoperative change in BCVA (in decimal) and complication rates. All postoperative endpoints were assessed on the last day of follow-up

\section{Surgical technique}

All patients underwent a three-port 25g PPV, using the oblique cannula insertion technique described previously[6], using a Constellation ${ }^{\circledR}$ vitrectomy machine (Alcon, Fort Worth, TX, USA), with an Ultravit ${ }^{\circledR}$ vitrectomy probe with a cutting rate of $5000 \mathrm{cuts} / \mathrm{min}$. Valved cannulas were used in all patients starting in August 2016. A Resight ${ }^{\circledR} 500$ (Zeiss, Germany) wide-angle visualization system was used to visualize the fundus. Cannulas were placed $3.5 \mathrm{~mm}$ posterior to the limbus in pseudophakic eyes and $4 \mathrm{~mm}$ posterior to the limbus in phakic eyes. A core vitrectomy was performed, followed by peripheral vitreous removal assisted by scleral indentation with light probe. Shaving of the vitreous base was performed around RB and suspicious lesions. Perfluorodecalin (Arcaline, Arcadophta, France) was used in some patients to immobilize a detached retina and facilitate peripheral vitreous removal. If an epiretinal 
membrane (ERM) or macular hole $(\mathrm{MH})$ was present in the macula, brilliant blue (Ocublue, Aurolab, India) dye was used and ERM and internal limiting membrane (ILM) peeling was performed. Perfluorodecalin was used in patients with a detached macula when membrane peeling was indicated. Fluid-air exchange with subretinal fluid (SRF) drainage through a peripheral RB was performed using a Charles Flute Cannula (Alcon, Fort Worth, TX, USA) or a vitrectomy probe. Perfluorodecalin was used or posterior retinotomies were performed to achieve complete SRF drainage in patients where the surgeon was concerned about the risk of a retinal fold forming in the macula. Posterior retinotomies were also performed in patients where pre-existing RB were not identified during vitrectomy. Perfluorodecalin was used and retinotomies were performed at the discretion of the surgeon. Complete SRF drainage was not required. Retinopexy of the margins of RB, lattice degenerations and other suspicious peripheral lesions were performed under air using an endolaser or cryotherapy probe. In some patients, retinopexy to the extent of the detached retina or a $360^{\circ}$ retinopexy was performed. The extent of retinopexy depended on the number and location of RB and the lattice degenerations and was also at the discretion of the surgeon. A non-expansive $20 \%$ mixture of sulfur hexafluoride (SF6) (Alchimia, Italy) or a $15 \%$ mixture of perfluoropropane (C3F8) (Alchimia, Italy) was used as a tamponade in all patients. The decision on which gas to use depended largely on the locations of RB. In patients with superior RB, $20 \%$ SF6 was generally used; in patients with inferior RB, 15\% C3F8 was generally used. However other factors were taken into consideration, like the presence and location of lattice degenerations and other suspicious peripheral lesions, patients' ability to posture after the operation etc. The final decision on the gas tamponade was made solely by the surgeon. After the air-gas exchange, cannulas were removed and the tightness of the sclerotomies was checked. If leakage was present, a digital massage of the sclerotomy was performed. If leakage persisted after digital massage, the sclerotomy was sutured using Vicryl 8-0 (Ethicon, Johnson \& Johnson Int). After all the cannulas were removed, digital palpation was used to check the IOP at the end of the operation. If the IOP was considered low, additional gas mixture was injected through the sclera using a 30-gauge needle. No scleral buckling was performed. Depending on the locations of break, patients were instructed on proper head positioning for the one-week period after the operation.

\section{Statistical analysis}

Quantitative variables - age and follow up period are given as means and standard deviations. BCVA is given as means and range. Retinal attachment success rates, lens status, state of the macula, extent of retinal detachment, and the number of patients with inferior RB in the ESG and ISG are given as numbers and percentages. To compare the age and follow-up periods between both groups of patients, the independent Student's t-test was calculated. To compare the BCVA before and after surgery, the paired Student's t-test was used for all three surgeons together and for each surgeon separately. To find differences in retinal attachment success rates, lens status, and state of the macula, the extent of retinal detachment and the number of patients with an inferior RB between the ESG and ISG was analyzed using contingency tables - the Pearson's chi-square test or in the case of small sample numbers, the exact Fisher test was calculated. Statsoft STATISTICA version 9 was used for statistical analysis. P-values less than 0.05 were considered to be statistically significant. 


\section{Results}

We examined the documentation of 407 PPV for retinal detachment performed on 368 eyes between October 2015 and June 2018 in the Department of Ophthalmology of the University Hospital Kralovske Vinohrady, Prague. 245 PPV were performed by the experienced surgeon, 162 by the inexperienced surgeons. The reason for exclusion from the study was the use of silicone oil tamponade in 53 PPV performed by the experienced surgeon and in 21 PPV performed by the inexperienced surgeons, other inclusion and exclusion criteria were not met in other cases. In the end, 216 eyes of 216 patients met all the inclusion and exclusion criteria and were included in the study, 106 in the ESG and 110 in the ISG. Table 1 shows the baseline demographic and clinical characteristics of the participants.

Table 1 - Baseline demographic and clinical characteristics of participants 


\begin{tabular}{|c|c|c|c|}
\hline & $\begin{array}{l}\text { Experienced surgeon } \\
\text { group }\end{array}$ & Inexperienced surgeon group & $\mathrm{P}$ \\
\hline Number of eyes & 106 & 110 & \\
\hline Number of patients (Male/Female) & $106(66 / 40)$ & $110(57 / 53)$ & \\
\hline Eye (Right/Left) & $54 / 52$ & $62 / 48$ & \\
\hline Age, mean (SD), years & $58.7( \pm 13.4)$ & $63.6( \pm 9.7)$ & 0.003 \\
\hline Mean preoperative BCVA (SD), decimal & $0.38( \pm 0,38)$ & $0.33( \pm 0.35)$ & 0.327 \\
\hline Artephakic (n, \%) & $34(32.1 \%)$ & $40(36.4 \%)$ & 0.507 \\
\hline Macula on $(\mathrm{n}, \%)$ & $40(37.7 \%)$ & $45(40.9 \%)$ & 0.633 \\
\hline RD extent, hours & 6.1 & 5.8 & 0.354 \\
\hline $\begin{array}{l}\text { Patients with inferior retinal break (n, } \\
\%)\end{array}$ & $41(38.7 \%)$ & $42(38.2 \%)$ & 0.940 \\
\hline Number of retinal breaks, mean (SD) & $2.9( \pm 2.8)$ & $2.5( \pm 2.2)$ & 0.173 \\
\hline Follow-up, mean (SD), months & $12.3( \pm 8.2)$ & $11.2( \pm 7.9)$ & 0.301 \\
\hline
\end{tabular}

$\mathrm{SD}$, standard deviation; BCVA, best-corrected visual acuity; RD, retinal detachment

One patient in the ESG underwent unsuccessful pneumatic retinopexy with laser barrage around the RB prior to the PPV, one patient had undergone scleral buckling for retinal detachment 11 years prior to the PPV. In the ISG two patients underwent unsuccessful laser retinopexy around the RB prior to the PPV. A macular hole was present preoperatively in 2 patients in the ESG. ILM peeling was performed during PPV for retinal detachment in both patients, the macular hole closed in one of them. Table 2 shows the surgical techniques and gas tamponade used in both groups.

Table 2 - Surgical techniques and tamponade selection 


\begin{tabular}{|l|l|l|l|}
\hline & Experienced surgeon group & Inexperienced surgeon group & P \\
\hline Use of perfluorodecaline, n, (\%) & $6(5.7 \%)$ & $10(9.1 \%)$ & 0.336 \\
\hline Retinotomy, n (\%) & $8(7.6 \%)$ & $6(5.5 \%)$ & 0.532 \\
\hline $360^{\circ}$ retinopexy (\%) & $12(11.3 \%)$ & $20(18.2 \%)$ & 0.156 \\
\hline Tamponade, C3F8 (\%)/ SF6 (\%) & $46(43.4 \%) / 60(56.6 \%)$ & $67(60.9 \%) / 43(39.1 \%)$ & $<0.001$ \\
\hline Sclerotomy suture, n (\%) & $1(0.9 \%)$ & & 0.020 \\
\hline
\end{tabular}

The single surgery success rate was achieved in 100 patients (94.3\%) in the ESG and 103 patients $(93.6 \%)$ in the ISG. The difference between both groups was not statistically significant $(P=0.828)$. Also, the differences between single surgery success rates between individual surgeons were not statistically significant. After the second surgery, the success rate was 100\% in the ESG and $97.3 \%$ in the ISG; a $100 \%$ success rate was achieved in ISG after the third surgery. The causes of failure were RB in the scar after cryopexy in 4 patients in the ESG and 2 patients in the ISG, reopening of the original RB in 1 patient in the ISG, a newly diagnosed RB in 3 patients in the ISG, and a PVR in 2 patients in the ESG and in 1 patient in the ISG.

Visual acuity improved from 0.38 decimal in the ESG and 0.33 decimal in the ISG to 0.73 decimal and 0.74 decimal, respectively. The difference was not statistically significant $(P=0.234)$.

The complication rate was similar between both groups. The most common complication was postoperative intraocular hypertension in $34(32.1 \%)$ patients in the ESG and $38(34.5 \%)$ patients in the ISG. Three patients in each group required temporary therapy with oral acetazolamide, and one patient in the ESG underwent laser iridotomy. All other cases were resolved with IOP-lowering topical medication. Intraocular hypotony occurred in one patient in each group and resolved without therapy. Two patients in the ESG and five patients in the ISG underwent further PPV for ERM formation in the macula. A postoperative macular hole occurred in two patients in the ESG and one patient in the ISG. Other complications occurred once in other patients from both groups. These included an iatrogenic posterior lens capsule tear, postoperative intraocular hemorrhage and subretinal perfluorocarbon. Cataract surgery was needed during the follow-up period in $66.7 \%$ of phakic patients in the ESG and $80.0 \%$ of phakic patients in the ISG. This difference was not statistically significant $(P=0.07)$.

\section{Discussion}


In our study, inexperienced surgeons were able to match the success rate of an experienced surgeon in uncomplicated RRD from the very start, suggesting a short learning curve. There is a possible bias in our study in the patient selection in each group. It may be argued that more complex cases would be operated by more experienced surgeon. We tried to minimize this bias by including only patients with no or only mild PVR. Only statistically significant difference between the two groups was in the age of the patients. We do not believe it influenced the results since all the other anatomical and functional characteristics of retinal detachment were similar in both groups. It should also be noted that $68 \%$ of all the PPV for retinal detachment performed by the inexperienced surgeons met all the inclusion and exclusion criteria and were included in the study. Single operation success rates in both groups were comparable to previously published figures for $25 \mathrm{~g}$ PPV with gas tamponade[10-13]. We believe this is due to the simplified operation technique used in our clinic. We try to limit the use of surgical techniques that have not been shown to improve uncomplicated RRD surgery outcomes. These include the use of perfluorodecalin[14, 15], complete subretinal fluid drainage[10, 16, 17], and $360^{\circ}$ retinopexy[18]. Limiting the use of these techniques may also prevent certain postoperative complications[19-22].

Surgical techniques employed by the experienced and surgeons in our study were similar, exceptions being the selection of intraocular tamponade and frequency of sclerotomy suturing. The inexperienced surgeons used C3F8 gas more often, which is longer lasting and more "secure" option. We believe this was due to inexperience a subsequent lack of self-confidence which led inexperienced surgeons to use C3F8 gas even in the cases where it might not have been necessary. In our opinion, the more frequent use of C3F8 in ISG was also the reason for higher cataract surgery rate after the PPV between the ESG and ISG, although the difference was not statistically significant. The higher rate of sclerotomy suturing could possibly be explained by the longer operating times in the ISG; however, we do not have data to support this claim as the duration of the operation was not recorded.

The complication rate was very similar in both study groups. There was a notable difference in cataract surgery rate after the PPV between the ESG and ISG, although the difference was not statistically significant The low postoperative intraocular hypotony rate can be explained by the oblique cannula insertion technique used by all surgeons $[23,24]$ and the use of digital palpation to assess IOP after the removal of cannulas.

Other studies have shown comparable results in PPV for RRD between experienced and inexperienced surgeons[25-30]. Ehrlich et al. and Dugas et al..[25,26] compared the success rates of sutureless PPV for RRD between experienced and inexperienced surgeons and used similar exclusion and inclusion criteria as our study. In both these studies, the combined single operation success rate for both studies was $75 \%$ (80.9\% and $75.4 \%$, respectively, for experienced surgeons and $70.0 \%$ and $74.8 \%$, respectively, for inexperienced surgeons), which was significantly lower than in our study. This can be explained by the improvements in surgical instruments and vitrectomy machines as well as in surgical techniques. For example, the high-speed vitrectomy has been shown to lower the number of iatrogenic retinal tears during PPV [31]. It should also be noted that in a study by Ehrlich et al., the less experienced vitrectomy surgeons 
were recruited from fellows who had extensive experience in other intraocular procedures whereas in our study, both inexperienced surgeons had no previous experience with intraocular surgery.

\section{Conclusions}

A 25g PPV with gas tamponade for treatment of RRD yields excellent anatomical results and improvement in BCVA. With good technique and use of modern vitrectomy machines and instruments, even inexperienced surgeons can achieve high single operation success rate, suggesting a short learning curve. The complication rate is comparable between experienced and inexperienced surgeons.

\section{Declarations}

\section{Data Availability}

Data are available from the corresponding author on reasonable request.

\section{Conflict of Interest}

The authors declare that they have no conflict of interest.

\section{Funding}

No funding was obtained for this study.

\section{Authors' contributions}

Study design, data acquisition and statistical analysis: MP, MV, ZS, JD, JV. Interpretation of data: MP, MV, PS. Writing (original draft): MP. Writing (review and editing): MP, MV, PS. Final approval of manuscript: all authors.

\section{References}

1. Hejsek L, Dusova J, Stepanov A, Rozsival P. Scleral buckling for Rhegmatogenous retinal detachment. Cesk Slov Oftalmol. 2014;70:110-3.

2. Chrapek O, Sin M, Jirkova B, Jarkovsky J, Rehak J. Functional Results of Cryosurgical Procedures in Rhegmatogenous Retinal Detachment Including Macula Region - Our Experience. Cesk Slov Oftalmol. 2013;69:202-6.

3. Chrapek O, Sin M, Jirkova B, Jarkovsky J, Rehak J. Anatomical Results of Cryosurgical Procedures in Rhegmatogenous Retinal Detachment - Our Experience. Cesk Slov Oftalmol. 2013;69:164-8.

4. Hilton GF, Grizzard WS. Pneumatic Retinopexy. Ophthalmology. 1986;93:626-41.

5. Escoffery RF, Joseph Olk R, Gilbert Grand M, Boniuk I. Vitrectomy Without Scleral Buckling for Primary Rhegmatogenous Retinal Detachment. Am J Ophthalmol. 1985;99:275-81. 
6. Veith M, Stranak Z, Pencak M, Vranova J, Studeny P. 25-Gauge Vitrectomy and Gas for the Management of Rhegmatogenous Retinal Detachment. Acta Univ Palacki Olomuc Fac Med. 2018;163:80-4.

7. McLeod D. Is It Time to Call Time on the Scleral Buckle? Br J Ophthalmol. 2004;88:1357-9.

8. Ho J-D, Liou S-W, Tsai C-Y, Tsai RJ-F, Lin H-C. Trends and Outcomes of Treatment for Primary Rhegmatogenous Retinal Detachment: A 9-Year Nationwide Population-Based Study. Eye. 2008;23:669-75.

9. Holladay JT. Visual acuity measurements. J Cataract Refract Surg. 2004;30:287-90.

10. Gotzaridis S, Liazos E, Petrou P, Georgalas I. 25-Gauge Vitrectomy and Incomplete Drainage of Subretinal Fluid for the Treatment of Primary Rhegmatogenous Retinal Detachment. Ophthalmic Surg Lasers Imaging Retina. 2016;47:333-5.

11. Miller DM, Riemann CD, Foster RE, Petersen MR. Primary Repair of Retinal Detachment With 25Gauge Pars Plana Vitrectomy. Retina. 2008;28:931-6.

12. Mura M, Tan HS, de Smet MD. Use of 25-Gauge Vitrectomy in the Management of Primary Rhegmatogenous Retinal Detachment. Retina. 2009;29:1299-304.

13. Kunikata H, Nishida K. Visual Outcome and Complications of 25-Gauge Vitrectomy for Rhegmatogenous Retinal Detachment; 84 Consecutive Cases. Eye. 2010;24:1071-7.

14. Vidne O, Blum Meirovitch S, Rabina G, Abd Eelkader A, Prat D, Barequet D, et al. Perfluorocarbon Liquid Vs. Subretinal Fluid Drainage during Vitrectomy for the Primary Repair of Rhegmatogenous Retinal Detachment: A Comparative Study. Curr Eye Res. 2018;43:1389-94.

15. Schneider EW, Geraets RL, Johnson MW. Pars Plana Vitrectomy Without Adjuvant Procedures for Repair of Primary Rhegmatogenous Retinal Detachment. Retina. 2012;32:213-9.

16. Chen X, Zhang Y, Yan Y, Hong L, Zhu L, Deng J, et al. Complete Subretinal Fluid Drainage Is Not Necessary During Vitrectomy Surgery for Macula-Off Rhegmatogenous Retinal Detachment with Peripheral Breaks: A Prospective, Nonrandomized Comparative Interventional Study. Retina. 2017;37:487-93.

17. Yamaguchi M, Ataka S, Shiraki K. Subretinal Fluid Drainage Via Original Retinal Breaks for Rhegmatogenous Retinal Detachment. Can J Ophthalmol. 2014;49:256-60.

18. Bilgin AB, Dogan ME, Aysun B, Apaydın KC. Pars plana vitrectomy with or without intraoperative $360^{\circ}$ peripheral endolaser for rhegmatogenous retinal detachment treatment. Int Ophthalmol. 2019;39:1687-94.

19. Cauchi P, Azuara-Blanco A, McKenzie J. Corneal Toxicity and Inflammation Secondary to Retained Perfluorodecalin. Am J Ophthalmol. 2005;140:322-3.

20. Wilbanks GA, Apel AJG, Jolly SS, Devenyi RG, Rootman DS. Perfluorodecalin Corneal Toxicity: Five Case Reports. Cornea. 1996;15:329-34.

21. Liu W, Gao M, Liang X. Management of Subfoveal Perfluorocarbon Liquid: A Review. Ophthalmologica. 2018;240:1-7. 
22. Bouheraoua N, Hrarat L, Parsa CF, Akesbi J, Sandali O, Goemaere I, et al. Decreased Corneal Sensation and Subbasal Nerve Density, and Thinned Corneal Epithelium as a Result of 360-Degree Laser Retinopexy. Ophthalmology. 2015;122:2095-102.

23. Bourgault S, Tourville E. Incidence of postoperative hypotony in 25-gauge vitrectomy: oblique versus straight sclerotomies. Canadian Journal of Ophthalmology. 2012;47:21-3.

24. Acar N, Kapran Z, Altan T, Unver YB, Yurtsever S, Kucuksumer Y. Primary 25-Gauge Sutureless Vitrectomy with Oblique Sclerotomies in Pseudophakic Retinal Detachment. Retina. 2008;28:106874.

25. Ehrlich R, Ahmad N, Welch S, Hadden P, Polkinghorne P. Vitreoretinal fellow surgical outcome of small gauge pars plana vitrectomy for acute rhegmatogenous retinal detachment. Graefes Arch Clin Exp Ophthalmol. 2011;249:1147-52.

26. Dugas B, Lafontaine P-O, Guillaubey A, Berrod J-P, Hubert I, Bron AM, et al. The Learning Curve for Primary Vitrectomy Without Scleral Buckling for Pseudophakic Retinal Detachment. Graefes Arch Clin Exp Ophthalmol. 2009;247:319-24.

27. Heimann H, Zou X, Jandeck C, Kellner U, Bechrakis NE, Kreusel K-M, et al. Primary vitrectomy for rhegmatogenous retinal detachment: an analysis of 512 cases. Graefes Arch Clin Exp Ophthalmol. 2006;244:69-78.

28. Sallam AB, Donachie PHJ, Yorston D, Steel DHW, Williamson TH, Jackson TL, et al. Royal College of Ophthalmologists' National Database Study of Vitreoretinal Surgery: Report 7, Intersurgeon Variations in Primary Rhegmatogenous Retinal Detachment Failure. Retina. 2018;38:334-42.

29. Mazinani BAE, Rajendram A, Walter P, Roessler GF. Does Surgical Experience Have an Effect on the Success of Retinal Detachment Surgery? Retina. 2012;32:32-7.

30. Keller J, Haynes RJ, Sparrow JM. Sequential Hypothesis Testing to Characterise the Learning Curve and Monitor Surgical Performance in Retinal Detachment Surgery. Ophthalmologica. 2016;235:15762.

31. Rizzo S, Genovesi-Ebert F, Belting C. Comparative Study Between a Standard 25-Gauge Vitrectomy System and a New Ultrahigh-Speed 25-Gauge System with Duty Cycle Control in the Treatment of Various Vitreoretinal Diseases. Retina. 2011;31:2007-13. 